\title{
Erratum
}

\section{Kraichnan Turbulence via Finite Time Averages ${ }^{\star}$}

\author{
C. Foias ${ }^{1,2}$, M. S. Jolly ${ }^{2}$ \\ 1 Department of Mathematics, Texas A\&M University, College Station, TX 77843, USA \\ 2 Department of Mathematics, Indiana University, Bloomington, IN 47405, USA. \\ E-mail: msjolly@indiana.edu
}

Received: 26 June 2007 / Accepted: 23 August 2007

Published online: 26 October 2007 - (C) Springer-Verlag 2007

Commun. Math. Phys. 255, 329-361 (2005)

To our regret, a number of misprints have occurred in [FJM]. The argument is correct, but the following typographical corrections should be made: in $(4.24) \sim$ should be $\gtrsim$, and the exponent on the logarithm should be $-3 / 2$. Two lines down, $>$ should be $\gtrsim$, and also the final $\tilde{\kappa}_{\eta}$ should be $\tilde{\eta}$. At the top of page 352, it is for (4.21) that we cannot provide a converse, and for (4.22) that we do find a weak converse. Accordingly, in the statement of Proposition $4.10, \lesssim$ should be $\gtrsim$. Consistent with this, $\lesssim$ should be $\gtrsim$ in (4.29), (4.30), (4.31), and in the last relation in the proof of Proposition 4.10. Missing in the right-hand side of (4.29) is the factor $1 / \psi(\kappa)$. In the last two relations on page 353, $\tilde{\kappa}_{\eta}^{1 / 3}$ should be $\tilde{\eta}^{1 / 3}$. There are other minor misprints, such as $\langle\cdot\rangle$ where there should be $\langle\cdot \tilde{\rangle}$, which can readily be corrected from the context.

\section{Reference}

[FJM] Foias, C., Jolly, M.S., Manley, O.P.: Kraichnan turbulence via finite time averages. Commun. Math. Phys. 255, 329-361 (2005)

Communicated by P. Constantin

The online version of the original article can be found under doi:10.1007/s00220-004-1274-5.

^ This work was partially supported by NSF grant number DMS-0511533. 